\title{
Correction of autofocusing errors due to specimen tilt for automated electron tomography
}

\author{
U. ZIESE, W. J. C. GEERTS, T. P. VAN DER KRIFT, \\ A. J. VERKLEIJ \& A. J. KOSTER \\ Molecular Cell Biology, Utrecht University, 3584 CH Utrecht, The Netherlands
}

Key words. Autofocus, automated electron tomography, bullfrog saccular hair cells, defocus measurement.

\begin{abstract}
Summary
Transmission electron microscopy images acquired under tilted-beam conditions experience an image shift as a function of defocus settings - a fact that is exploited as a method for defocus determination in most of the automated tomography data collection systems. Although the method was shown to be highly accurate for a large variety of specimens, we point out that in its original design it can strictly only be applied to images of untilted samples. The application to tilted samples and thus in automated electron tomography is impaired mainly due to a defocus change across the images, resulting in reduced accuracy. In this communication we present a method that can be used to improve the accuracy of the basic autofocusing procedures currently used in systems for automated electron tomography.
\end{abstract}

\section{Introduction}

Electron tomography is a versatile method for obtaining threedimensional (3D) information of biological and materials sciences samples (100-500 nm thick) with 2-20 nm resolution by transmission electron microscopy (TEM) (Frank, 1992). Electron tomography is generally applicable to isolated particles (e.g. macromolecules) as well as to pleomorphous structures like the Golgi complex or other cellular organelles, whole cells or tissue sections. For recent reviews on electron tomography see the papers by Baumeister et al. (1999) and McEwen \& Marko (2001).

The basic concept of electron tomography was outlined in the late 1960s (DeRosier \& Klug, 1968; Hart, 1968; Hoppe et al., 1968; Crowther et al., 1970) but a number of technical obstacles, concisely described in the mid-1970s (Hoppe et al., 1976, Hoppe \& Hegerl, 1980), prevented realization of its potential until the mid-1990s. The basic procedure of electron tomography consists of two steps: data collection and data processing. For data collection, a series of two-dimensional (2D)

Correspondence: A. J. Koster. Tel.: +31 30253 7763; E-mail: a.j.koster@bio.uu.nl projection images of a specimen tilted over a wide angular range is acquired (so-called tilt series). Typically, a tilt series consists of 50-150 digital images (recorded with a CCD camera) taken over an angular range of $-70^{\circ}$ to $+70^{\circ}$. After data collection, a reconstruction step is carried out that comprises alignment of the projection images and 3D reconstruction typically via filtered back-projection. For a description of 3D electron microscopy, including electron tomography and some aspects of the data processing involved, the reader is referred to Baumeister \& Steven (2000).

In the mid-1990s, data collection systems became available (Dierksen et al., 1992, 1993; Koster et al., 1992, 1997; Grimm et al., 1997; Rath et al., 1997) that were capable of collecting a tilt series in an automated fashion. To collect a useful tilt series, two conflicting requirements need to be reconciled. First, a tilt series has to be recorded that covers as wide an angular range as possible in as many increments as possible. Second, the electron dose has to be kept low to avoid radiation damage of the specimen. Owing to the mechanical movement of the specimen when the stage is tilted, the focus and image position will change during collection of a tilt series. With the advent of computer-controlled microscopes and large-area digital cameras (CCD cameras), it became possible to implement automated procedures for the repetitive (50-150 times) measurement and correction of (a) defocus (autofocus) (Koster et al., 1987, 1989) and (b) image shifts (autotracking). Today, automated data collection software packages, which correct for the movement of the specimen during data collection, are available both commercially and within the scientific community.

During the last 5 years we have applied electron tomography in our laboratory as a tool for 3D structural investigations of both biological and materials sciences samples (e.g. plastic-embedded and stained sections, $100-300 \mathrm{~nm}$ thick; negatively stained isolated structures, 50-100 nm in diameter; frozen-hydrated viruses and tubular structures; zeolite particles, 50-200 nm).

We observed that the automatic focusing at higher tilt angles $\left(>50^{\circ}\right)$ was often inaccurate. The variability in focusing accuracy became more apparent after developing the precalibration 
data collection approach (Ziese et al., 2002), in which the image shifts and focus changes are measured (calibrated) as a function of specimen tilt prior to data collection. Though the focus generally changed smoothly with specimen tilt, as expected, occasionally large focus changes were measured at the higher tilt angles. These errors could be several micrometres in magnitude, which is sufficiently large to limit the resolution seriously and hamper reliable interpretation of a tilt series.

In this communication we propose a significant modification of the autofocus method as it is currently implemented in practically all of the available automated data collection systems. The modified method is designed to work on highly tilted specimens and is suitable for automatic focusing as part of an automated procedure to collect tilt series. At the higher tilt angles the modified method performs more reliably than without a correction for specimen tilt.

\section{Beam-tilt autofocus method}

With the tilted-beam method two images are taken, each acquired with a different beam tilt. Figure 1 shows a schematic diagram of the projection of an untilted specimen, with a certain defocus $D_{0}$ and beam tilt $\vec{\beta}$.

In comparison with an image taken without beam tilt, the image will be shifted by

$$
\vec{s}=D_{0} * \tan (\vec{\beta})
$$

For a known beam tilt it is therefore possible to determine the defocus by measuring the image shift that is given by the crosscorrelation of two images acquired with different beam tilts. For the sake of clarity, the effects of astigmatism, misalignment as well as beam-tilt induced defocus are not taken into account in Eq. (1).

Although the beam-tilt method has shown to be highly accurate for untilted specimens (5-50 nm; Koster et al., 1989; Dierksen et al., 1993), the method can be far less accurate for

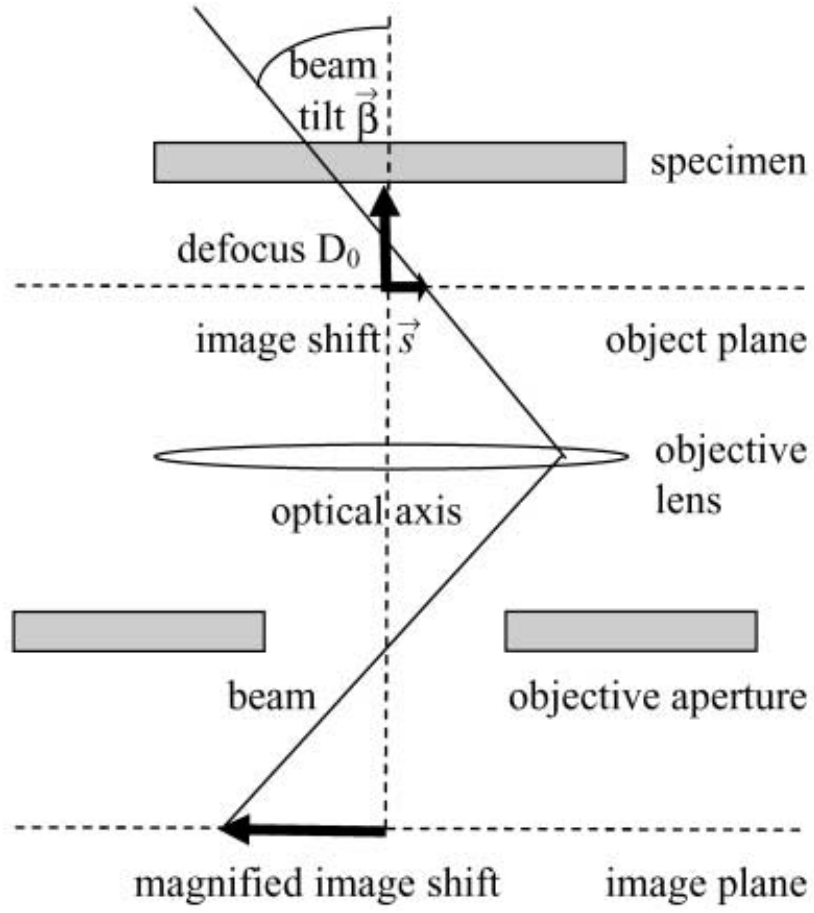

Fig. 1. Schematic diagram of defocus $D_{0}$ and beam-tilt $\beta$ induced image shift $\vec{s}$ of the projection of an untilted specimen.

tilted specimens. Figure 2 shows the projection images of a Epon-embedded section of a bullfrog saccular hair cell at tilt angles of $-70^{\circ}, 0^{\circ}$ and $0^{\circ}$. Whereas the whole area of $3.2 \mu \mathrm{m}$ $\times 3.2 \mu \mathrm{m}$ of the image displays the same defocus at $0^{\circ}$, it can be seen that the defocus varies across the image at $-70^{\circ}$ (right side blurry) and $+70^{\circ}$ (left side blurry). The defocus difference between the edge and the centre of the image is $4.4 \mu \mathrm{m}$ $\left[3.2 \mu \mathrm{m} / 2 \times \tan \left(70^{\circ}\right)\right]$ for the tilted views.

Defocus determination by the beam-tilt method for tilted samples is thus impaired by an elongation of the cross-correlation peak, which has contributions from areas with different defocus
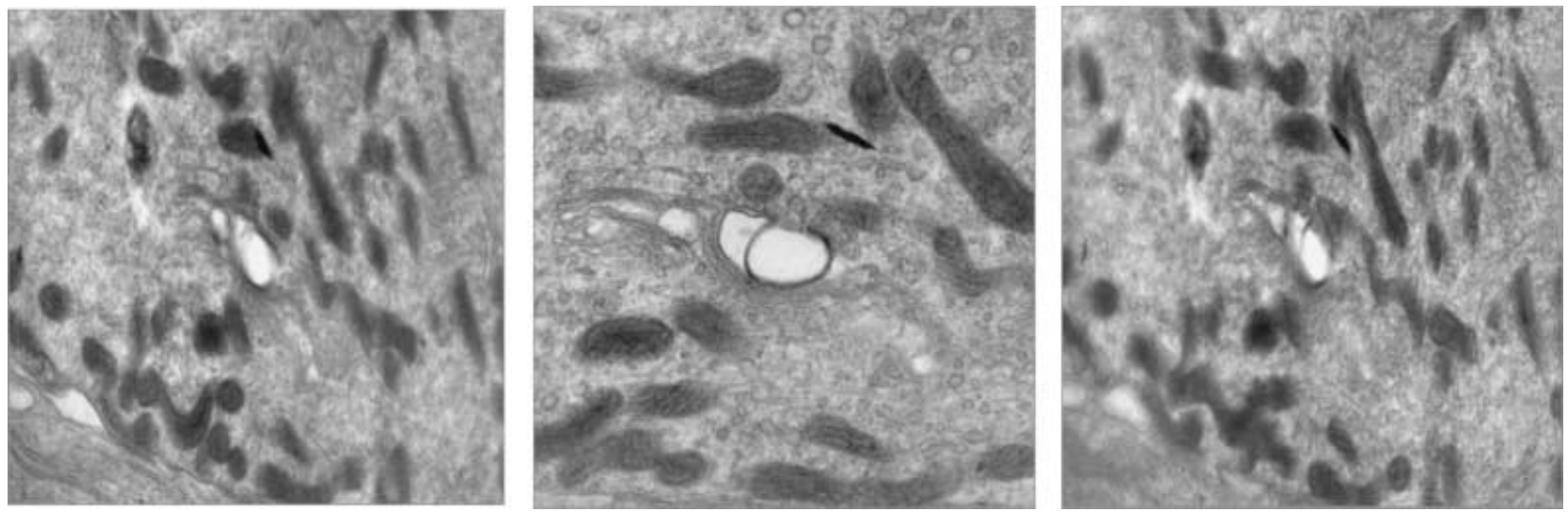

Fig. 2. Projection images of an Epon-embedded section of a bullfrog saccular hair cell at tilt angles of $-70^{\circ}$ (left), $0^{\circ}($ middle, $3.2 \mu \mathrm{m} \times 3.2 \mu \mathrm{m})$ and $+70^{\circ}$ (right). The defocus was about $-10 \mu \mathrm{m}$ in the centre of the images but varies across the tilted images by $8.8 \mu \mathrm{m}$ (images are partly blurred). 

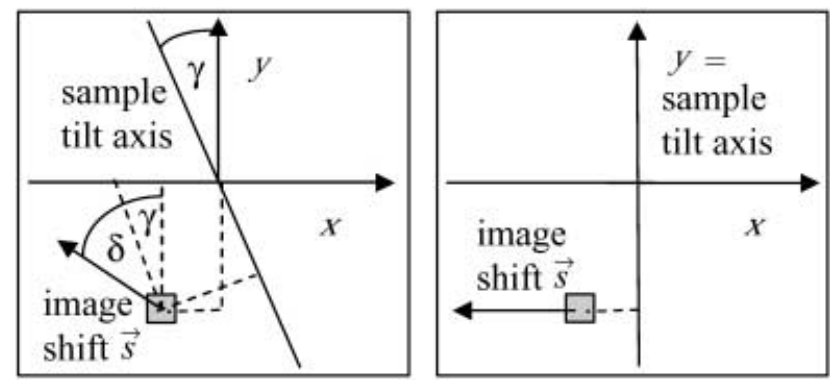

Fig. 3. General geometry of beam-tilt-induced image shift in the image $x y$ plane (left). The angle between the sample-tilt axis and the image $y$-axis is $\gamma$ and the angle between the image shift $\vec{s}$ and the tilt axis is $\delta$. The righthand side shows the special case when the sample-tilt axis is parallel to the $y$-axis and the image shift is parallel to the $x$-axis as discussed in this paper.

values. In an unfavourable case, the autofocus procedure could be biased towards a high-contrast region near the edge of the field of view and, consequently, measure a defocus value several micrometres different from the defocus present in the centre of the image. At lower magnifications the field of view of the CCD camera will be relatively large - resulting in a large error in defocus measurement. The problem should therefore be kept in mind especially if the defocus is determined at a lower magnification than that used for image acquisition as is possible, for example, with precalibration electron tomography (Ziese et al., 2002) or with large-area $(2 \mathrm{k} \times 2 \mathrm{k}$ and $4 \mathrm{k} \times 4 \mathrm{k}$ pixel) CCD cameras.

In this communication we examine the location of image features-assuming a sample geometry of an extended slab-under tilted-sample, tilted-beam conditions and present a method that improves the defocus measurement under these conditions.

\section{Modification of the beam-tilt method for tilted specimens}

Figure 3(left) shows the general geometry of a beam-tilt-induced image shift $\vec{s}$ in the image $x y$-plane. The angle between the sample-tilt axis and the image $y$-axis is $\gamma$ and the angle between the image shift $\vec{s}$ as induced by the beam tilt and the tilt axis is $\delta$. For the further discussion, however, we assume that the sample-tilt axis goes through the centre of the image and is parallel to the image $y$-axis $\left(\gamma=0^{\circ}\right)$. The image is assumed to be in focus along the tilt axis and the image shift $\vec{s}$ is assumed to be parallel to the $x$-axis $\left[\delta=90^{\circ}, s_{y}=0, \vec{s}=s_{x}\right.$; Fig. 3(right)]. Figure 4 displays the location of image features of a tilted sample with and without beam tilt $\beta$. The upper row shows the positions in the $x z$-plane and the middle row in the $x y$-plane.

When a tilted sample is imaged, the defocus $D$ of an image feature depends on the $x$-coordinate and the sample-tilt angle $\alpha$ :

$$
D=x * \tan (\alpha)
$$

From Eqs (1) and (2) it follows that the image shift $\vec{s}$ of such a feature will be

$$
\vec{s}=x * \tan (\alpha) * \tan (\vec{\beta}) .
$$

Thus, the further the feature is away from the centre of the image - and thus away from the focus plane - the larger the image shift will be. Furthermore, the image shift will be positive for positive defocus and negative for negative defocus, when the beam tilt is in one direction and the reverse for a beam tilt in the opposite direction. The bottom row of Fig. 4 shows the image shifts that will be indicated by the crosscorrelation of different areas of the beam-tilted and the not beam-tilted images of the sample, displayed as vector fields.

Therefore, to ensure that all areas of the images give rise to the same cross-correlation peak, it is necessary to squeeze/ stretch the $\pm \beta$ beam-tilted images parallel to the $x$-axis and with respect to the $y$-(sample-tilt) axis:

$$
\operatorname{stretch}(\alpha, \pm \beta)=1+\tan (\alpha) * \tan ( \pm \beta) \text {. }
$$

If the beam-tilt angle is not known, it can be calibrated from the image shift measurement of an untilted sample for a known defocus using Eq. (1).

From Eq. (4) the relative stretch between the $+\beta$ and $-\beta$ beam-tilted images can be calculated:

$$
\begin{aligned}
\text { rel.stretch } & =\frac{\text { stretch }(\alpha+\beta)}{\text { stretch }(\alpha-\beta)} \\
& =\frac{1+\tan (\alpha) * \tan (\beta)}{1-\tan (\alpha) * \tan (\beta)}=\frac{\cos (\alpha-\beta)}{\cos (\alpha+\beta)}
\end{aligned}
$$

Note that this equation is the same as the cosine stretch for the cross-correlation of two images acquired at $(\alpha-\beta)$ and $(\alpha+\beta)$ sample-tilt angles (Guckenberger, 1982). It can be interpreted in the way that an image acquired under a certain sampletilt angle $\alpha$ and beam-tilt angle $\beta$ is the same as one acquired under a sample-tilt angle $(\alpha-\beta)$.

In the general case, when neither the sample-tilt axis is parallel to the $y$-axis nor the beam-tilt-induced image shift is in a direction perpendicular to the $y$-axis $\left(\gamma \neq 0^{\circ}, \delta \neq 90^{\circ}\right.$ and $\delta \neq 270^{\circ}$ ), the image transformation can be determined by substituting $x$ in Eq. (3) with the distance between the sample feature and the sample-tilt axis. Figure 5 depicts the special case when $\gamma=0^{\circ}$ and $\delta=0^{\circ}$, which is equivalent to an image shear.

Figure 6 compares the contributions of different types of errors in defocus determination for different magnifications at a sample-tilt angle of $60^{\circ}$. The theoretical comparison assumes an experimental set-up with a CCD camera with $2048 \times 2048$ pixels with a size of $14 \mu \mathrm{m}$, a post-magnification of $1.8 \times$ and a beam tilt of $4 \mathrm{mrad}$. The figure includes values for the calculated errors caused by: (a) a broadening of the peak due to a sample thickness of $100 \mathrm{~nm}$ [error $=100 \mathrm{~nm} / 2$ / $\left.\cos \left(60^{\circ}\right)\right]$, which is the same for all magnifications; (b) a limited accuracy in the measurement of the location of the XCF peak of 1 pixel $[\operatorname{error}($ magnification $)=14 \mu \mathrm{m} /$ magnification $/$ postmagnification $\left.\times \tan \left(70^{\circ}\right)\right]$; and the use of $(c)$ only the central 

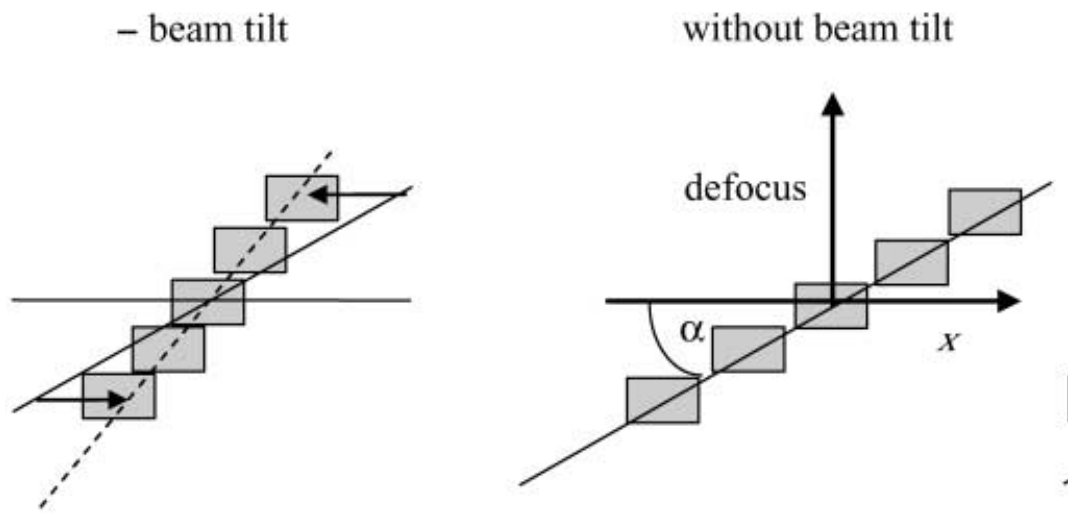

+ beam tilt
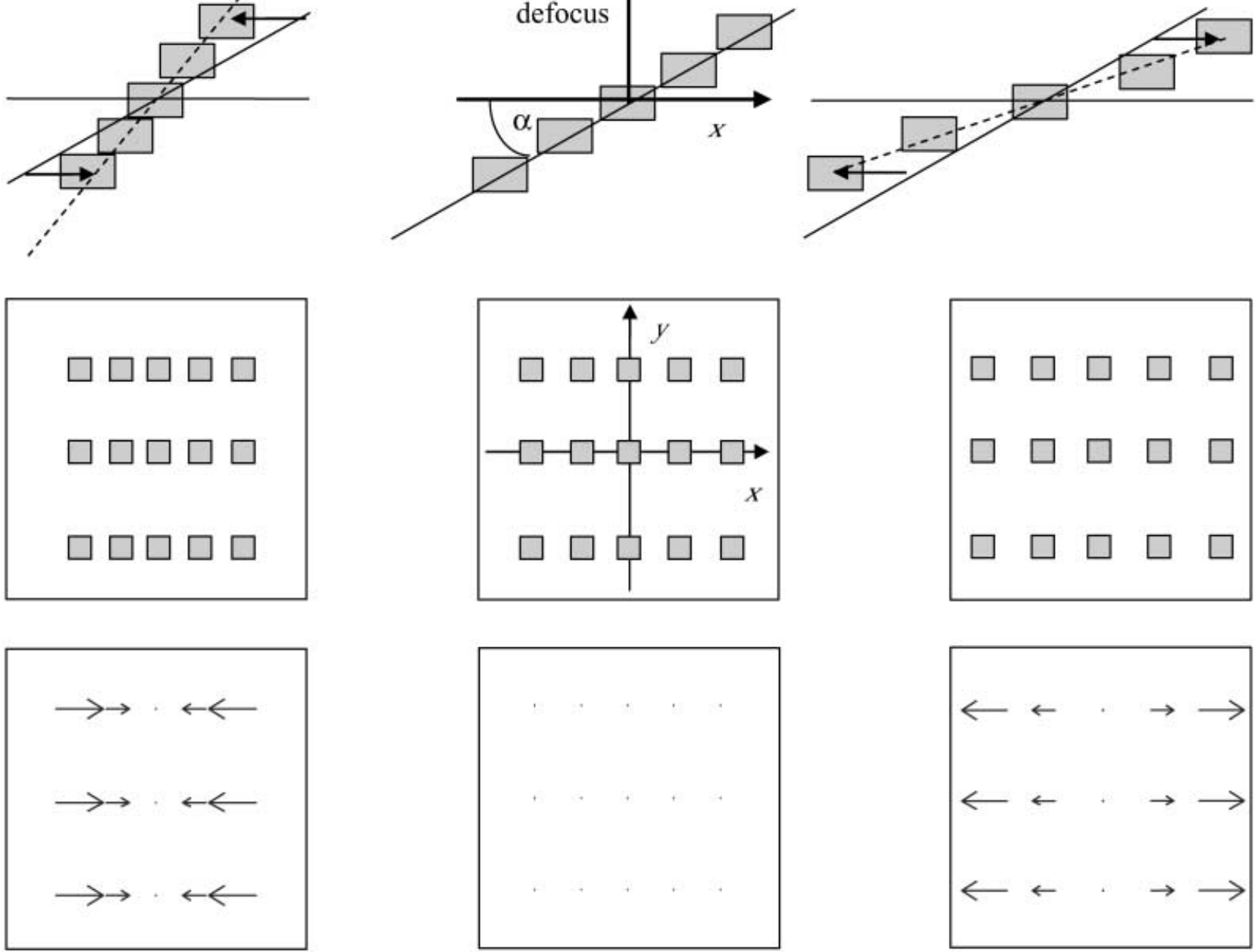

Fig. 4. Location of image features of a tilted sample without and with beam-tilt $\beta$. The upper row shows the positions in the $x z$-plane and the middle row in the $x y$-plane. The bottom row shows the image shifts that will be indicated by the cross-correlation of different areas of the beam-tilted and the not beamtilted images of the sample, displayed as vector fields.

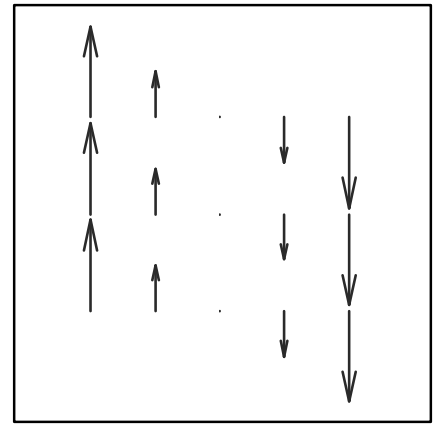

Fig. 5. Image shift vectors of a tilted sample for the special case when both sample-tilt axis and image shifts are parallel to the image $y$-axis. The image appears 'sheared'.
$25 \%$ [error(magnification $)=2048 / 2 \times 0.25 \times($ b) $]$ or $(\mathrm{d})$ an additional $75 \%$ [and thus $100 \%$, error(magnification) $=$ $2048 / 2 \times$ (b)] of the beam-tilted images for cross-correlation. The use of only a central part of the images is routinely done in programs for the automated acquisition of electron tomography tilt series to improve defocus determination. However, in spite of these attempts, the errors can still be as large as several micrometres at high tilt angles and low magnifications, when no correction for specimen tilt is taken into account.

The possible errors in defocus determination from (c) and (d) above due to the defocus ramp within the two images acquired for automatic focusing will - theoretically - be completely avoided by stretching the two images by an amount given by Eqs (4) or (5). Therefore, we strongly recommend that stretching is incorporated into existing data collection systems 


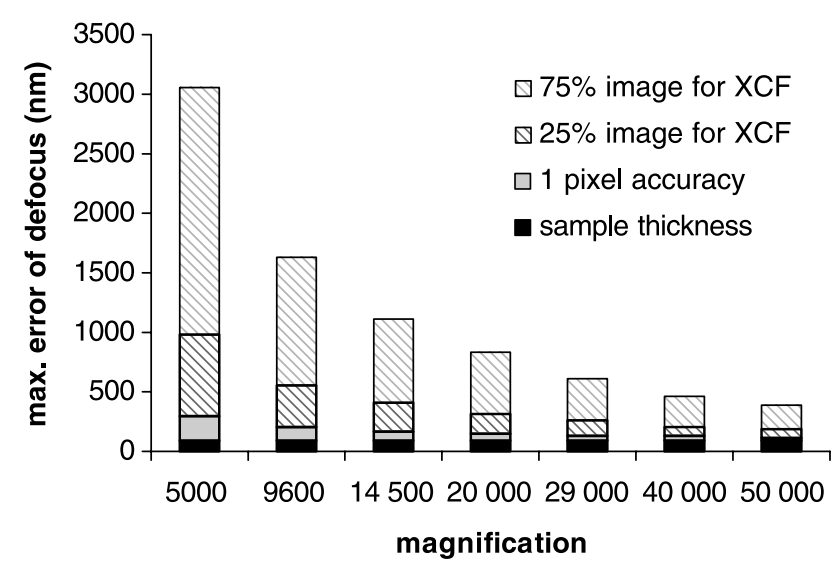

Fig. 6. Comparison of the contributions of different types of errors in defocus determination for a sample-tilt angle of $60^{\circ}$ and a beam tilt of 4 mrad at different magnifications (see text for details).

because it is an essential modification for accurate beam-tiltbased autofocusing methods. The practical achievable accuracy in the presented correction depends on the accuracy to which the sample-tilt angle (due to a possible-but unknownpretilt of the sample) and the beam-tilt angle (due to misalignment or reproducibility in the direction of the beam) are known. If we assume for instance that the sample-tilt angle is known to an accuracy of $10^{\circ}$, then the suggested correction will only be beneficial for sample-tilt angles greater than that value.

\section{Experimental verification}

To evaluate the improvement in defocus determination of tilted samples by the beam-tilt method after application of the presented formulas, we acquired two sets of images on a 200kV Tecnai 20 transmission electron microscope (FEI Co., Eindhoven, The Netherlands) with a bottom-mounted slow-scan CCD camera (TemCam F214, Tietz Video and Image Processing Systems, TVIPS GmbH, Gauting, Germany). The camera is composed of $2048 \times 2048$ square pixels of $14 \mu \mathrm{m}$. Image acquisition was performed under remote control by either a JavaScript macro in TIA (FEI Co.) or a TCL macro in EMMENU (TVIPS $\mathrm{GmbH}$ ), which communicated with the microscope via the Tecnai Scripting software (FEI Co.).

For the first set of images we used beam-tilt values of $T_{x}=$ 0.02000 and $T_{y}=0.02038$ (the ' + ' beam tilt), and $T_{x}=-0.0200$ and $T_{y}=-0.02038$ (the '-' beam tilt), for the second set the beamtilt values were $T_{x}=0.04000, T_{y}=0.04076$ and $T_{x}=-0.0400$, $T_{y}=-0.04076$. The named values are in arbitrary units (as indicated by the Tecnai Scripting software) and gave rise to image shifts almost parallel to the $x$-axis of the CCD images. The shifts were therefore also almost perpendicular to the sample-tilt axis that was nearly parallel to the CCD image $y$-axis (about $6^{\circ}$ variance).

The first set of images investigates the improvement in defocus determination at different sample-tilt angles. We acquired images for '+' beam-tilt and '-' beam-tilt illumination conditions for sample-tilt angles $\alpha$ of $-56^{\circ},-40^{\circ},-20^{\circ}, 0^{\circ},+20^{\circ}$, $+40^{\circ}$ and $+56^{\circ}$. All images were taken at the same position of a calibration grid (Electron Microscopy Sciences, Washington, PA) at a nominal magnification of $5000 \times$ (the calibrated pixel size was $3.1 \mathrm{~nm}$ with a post-magnification of $1.8 \times$ and binning 2 ) representing a specimen area of $3.2 \times 3.2 \mu \mathrm{m}$.

The second set of images investigates the improvement in defocus determination at different magnifications. We acquired images for '+' beam-tilt and '-' beam-tilt illumination conditions for a sample-tilt angle $\alpha$ of $+60^{\circ}$. Images were taken at 20 different positions of a section of bullfrog saccular hair cell stereocilia at nominal magnifications of 5000×, 9600×, $14500 \times$ and $19000 \times$, representing specimen areas of $3.2 \times 3.2,1.7 \times$ $1.7,1.1 \times 1.1$ and $0.8 \times 0.8 \mu \mathrm{m}$.

Image processing was performed with MATLAB (The MathWorks, Inc., Natick, MA) on a PC running under Windows XP (Microsoft Co., Redmond, WA).

\section{Results and discussion}

For comparison, we extracted nine small areas of a size of $128 \times$ 128 pixels from the $1024 \times 1024$-pixel images, which we had taken for '+' and '-' beam-tilt illumination conditions (Fig. 7).

Figure 8 displays the image shifts and cross-correlation functions (XCFs) of the first set of images that we have taken for the sample-tilt angles of $-56^{\circ},-40^{\circ},+40^{\circ}$ and $+56^{\circ}$ (from left to right) at a nominal magnification of 5000×. The first row shows the image shifts between the corresponding areas

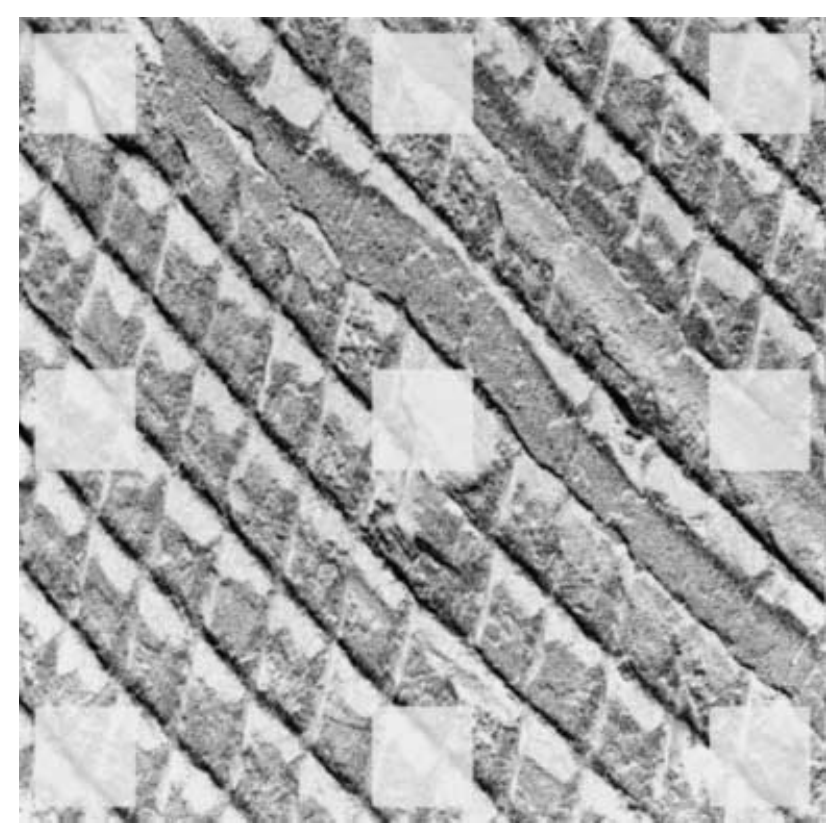

Fig. 7. One of the $1024 \times 1024$-pixel $(3.2 \mu \mathrm{m} \times 3.2 \mu \mathrm{m})$ images of a calibration grid that were used to evaluate the improvement in defocus determination of tilted samples by the method presented in this paper. The overlay indicates the $128 \times 128$-pixel-sized areas from which the shifts between the '+' and '-' beam-tilt images were calculated. 

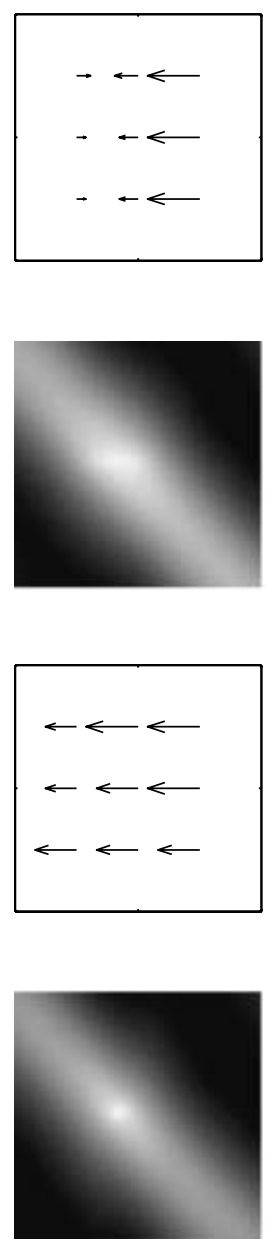
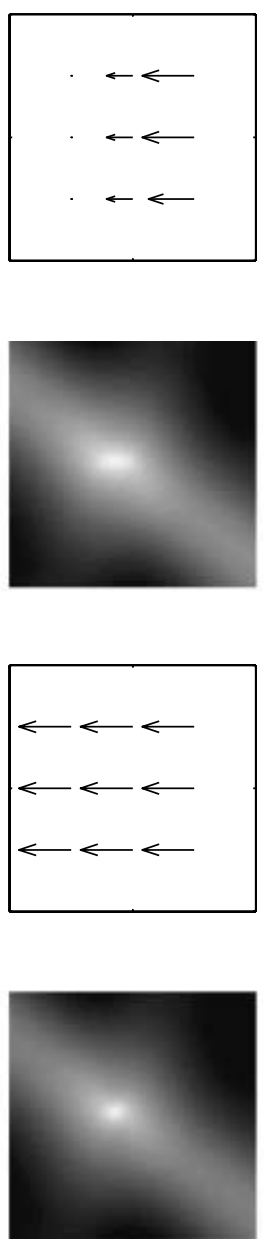
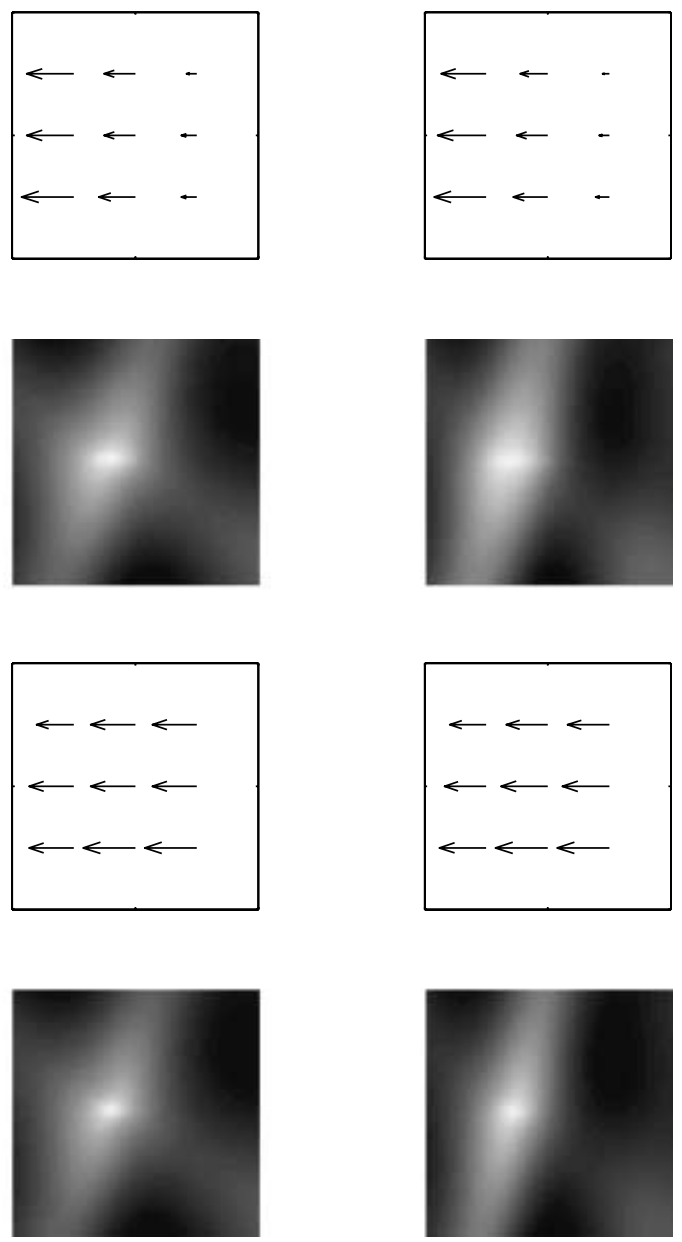

Fig. 8. Image shifts between corresponding areas (see Fig. 6) for ' + ' and '-' beam-tilt images for sample-tilt angles of $-56^{\circ},-40^{\circ},+40^{\circ}$ and $+56^{\circ}$ (from left to right) without (first row) and with (third row) correction for sample tilt. The second and fourth rows show the central part of the cross-correlations that were calculated from the full images of the uncorrected and corrected images. The broadening of the cross-correlation peak is less severe in the corrected case.

for '+' and '-' beam-tilt images, which are calculated via crosscorrelation and displayed as vector fields. All image-shift vectors for all tilt angles are perpendicular to the image $y$-axis and their size and orientation depends on the $x$-coordinate inside the images. Comparison with the displacement maps of Fig. 4 shows that this is exactly what was expected (when the centre of the images was not in focus). The third row of Fig. 8 shows the displacement fields after correction. The fourth row shows the central part of the XCF that was calculated from the entire images. The broadening of the cross-correlation peak is less severe than in the uncorrected case (second row of Fig. 8). The correction was achieved by manually adjusting the relative stretch values until the differences in the shift vectors for all nine test areas disappeared for all angles. A linear leastsquares fit of the tangents of sample-tilt angles $\alpha\left(-56^{\circ},-40^{\circ}\right.$, $-20^{\circ},+20^{\circ},+40^{\circ}$ and $+56^{\circ}$ ) according to (rel.stretch -1$) /$ $($ rel.stretch +1$)=\tan (\beta) \times \tan (\alpha)$, which is a modification of Eq. (5), gave a beam-tilt angle $\beta$ of $5 \pm 0.6 \mathrm{mrad}$.
Figure 9 compares the specimen-tilt-induced image shifts/ autofocusing errors at different magnifications (5000×, 9600×, $14500 \times$ and $19000 \times$ ) for the second set of images. The sample was a typical heavy-metal-stained, plastic-embedded section of biological material (bullfrog saccular hair cell stereocilia). We acquired images for '+' beam-tilt and '-' beam-tilt illumination conditions for a sample-tilt angle $\alpha$ of nominal $+60^{\circ}$ at 20 different positions on the sample. The $x$-image shifts between the corresponding areas for ' + ' and '-' beam-tilt images were calculated and the differences in shift between the areas at the left side from that at the right side of the images were determined (three per image pair and thus 60 at each magnification) and divided by 2 . The average shift values (Fig. 9, light grey bars) display the same dependence on the magnification as the theoretical values for the maximum sample-tilt-induced autofocusing errors (Fig. 6). This is what is expected as there is a linear relationship between the values (Eq. 1). Standard deviations of the measurement are quite 


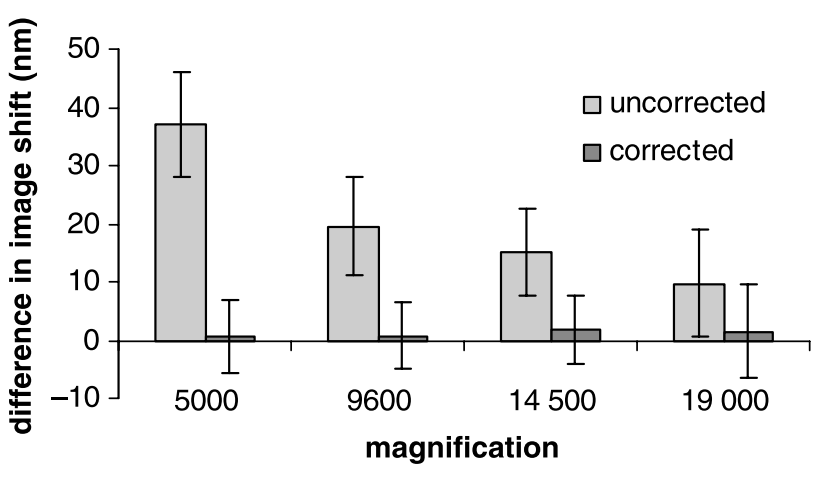

Fig. 9. Measured average image shift differences for magnifications of $5000 \times, 9600 \times, 14500 \times$ and $19000 \times$ without (light grey bars) and with (dark grey bars) correction for sample-tilt. The average shift values after correction are negligible.

large and can be explained by the combined effect of additional autofusing errors (Fig. 6) and a divergence of pretilt among the 20 measurement positions on the sample grid. The average shift values after correction for a sample-tilt angle of $\alpha=+58^{\circ}$ (Fig. 9, dark grey bars) are negligible.

\section{Conclusions}

We have modified the defocus measurement method based upon beam-tilt-induced image shifts to be suitable for automated electron tomography. This is necessary because defocus variations across the sample significantly degrade the accuracy of current beam-tilt autofocus methods, giving unreliable measurements. We have shown that the problem can be overcome by stretching the beam-tilted images prior to crosscorrelation by a factor that can be derived from four parameters of the data collection set-up: (a) the orientation of the sample-tilt axis, (b) the sample-tilt angle $\alpha$, (c) the orientation (direction) of the beam-tilt axis and (d) the beam-tilt angle $\beta$. In the case that the sample-tilt axis is parallel to the image $y$-axis and the beam-tilt-induced image shift is parallel to the $x$-axis, the required stretch would be

$$
\operatorname{stretch}(\alpha, \pm \beta)=1+\tan (\alpha) * \tan ( \pm \beta) \text {. }
$$

We emphasize that even if the problem might not be directly visible to the human eye by looking at the original images (the stretch is, for example, just 1.007 for $\alpha=60^{\circ}$ and $\beta=4 \mathrm{mrad}$ ), it can lead to relatively large errors in defocus determination (several micrometres). We suggest that the correcting stretch should be routinely applied when using the beam-tilt method to determine the defocus for automated electron tomography.

\section{Acknowledgements}

We would like to thank Manfred Auer (Skirball Institute of Biomolecular Medicine, NYU, and The Rockefeller University) and Jim Hudspeth (HHMI and The Rockefeller University) for sections of bullfrog saccular hair cell stereocilia and David Agard (Macromolecular Structure Group, UCSF) for critical reading of the manuscript. The research of U.Z. and A.J.K. (fellowship) has been made possible by support of the Royal Netherlands Academy of Arts and Sciences (KNAW).

\section{References}

Baumeister, W., Grimm, R. \& Walz, J. (1999) Electron tomography of molecules and cells. Trends Cell Biol. 9, 81-85.

Baumeister, W. \& Steven, A.C. (2000) Macromolecular electron microscopy in the era of structural genomics. Trends Biochem. Sci. 25, 624-631.

Crowther, R.A., DeRosier, D.J. \& Klug, A. (1970) The reconstruction of a three-dimensional structure from its projections and its application to electron microscopy. Proc. R. Soc. Lond. (A), 317, 319-340.

DeRosier, D.J. \& Klug, A. (1968) Reconstruction of three-dimensional structures from electron micrographs. Nature, 217, 130-134.

Dierksen, K., Typke, D., Hegerl, R. \& Baumeister, W. (1993) Towards automatic electron tomography. II. Implementation of autofocus and low-dose procedures. Ultramicroscopy, 49, 109-120.

Dierksen, K., Typke, D., Hegerl, R., Koster, A.J. \& Baumeister, W. (1992) Towards automatic electron tomography. Ultramicroscopy, 40, 71-87. Frank, J. (1992) Electron Tomography. Plenum Press, New York.

Grimm, R., Barmann, M., Hackl, W., Typke, D., Sackmann, E. \& Baumeister, W. (1997) Energy filtered electron tomography of iceembedded actin and vesicles. Biophys. J. 72, 482-489.

Guckenberger, R. (1982) Determination of a common origin in the micrographs of tilt series in three-dimensional electron microscopy. Ultramicroscopy, 9, 167-174.

Hart, R. (1968) Electron microscopy of unstained biological material: the polytropic montage. Science, 159, 1464-1467.

Hoppe, W. \& Hegerl, R. (1980) Three-dimensional structure determination by electron microscopy (nonperiodic specimens). Computer Processing of Electron Microscope Images (ed. by P. W. Hawkes), pp. 127-185. Springer, Berlin.

Hoppe, W., Langer, R., Knesch, G. \& Poppe, C.H. (1968) Proteinkristallstrukturanalyse mit Elektronenstrahlen. Naturwissenschaften, 5, 333-336.

Hoppe, W., Schramm, H.J., Sturm, M., Hunsmann, N. \& Gassmann, J. (1976) Three-dimensional electron microscopy of individual biological objects. Part I. Meth. Z. Naturforsch. 31a, 645-655.

Koster, A.J., Chen, H., Sedat, J.W. \& Agard, D.A. (1992) Automated microscopy for electron tomography. Ultramicroscopy, 46, 207-227.

Koster, A.J., de Ruiter, W.J., van den Bos, A. \& van der Mast, K.D. (1989) Autotuning of aTEM using minimum dose. Ultramicroscopy, 27, 251-272.

Koster, A.J., Grimm, R., Typke, D., Hegerl, R., Stoschek, A., Walz, J. \& Baumeister, W. (1997) Perspectives of molecular and cellular electron tomography. J. Struct. Biol. 120, 276-308.

Koster, A.J., van den Bos, A. \& van der Mast, K.D. (1987) An autotuning method for a TEM. Ultramicroscopy, 21, 209-222.

McEwen, B.F. \& Marko, M. (2001) The emergence of electron tomography as an important tool for investigating cellular ultrastructure. J. Histochem. Cytochem. 49, 553-563.

Rath, B.K., Marko, M., Radermacher, M. \& Frank, J. (1997) Low-dose automated electron tomography: a recent implementation. J. Struct. Biol. 120, 210-218.

Ziese, U., Janssen, A.H., Murk, J.L., Geerts, W.J.C., Krift, T., Verkleij, A.J. \& Koster, A.J. (2002) Automated high-throughput electron tomography by pre-calibration of image shifts. J. Microsc. 205, 187-200. 